\title{
PROPUESTA METODOLÓGICA PARA EL ANÁLISIS DE LA EVIDENCIALIDAD EN EL DISCURSO CIENTÍFICO
}

\author{
PROPOSAL OF A METHODOLOGY FOR ANALYSING \\ EVIDENTIALITY IN SCIENTIFIC DISCOURSE
}

\author{
Dorota Kotwica ${ }^{1}$ \\ Universidad Pedagógica de Cracovia \\ Grupo Val.Es.Co. \\ dorota.kotwica@up.krakow.pl
}

Recibido: 20/12/2016

Aceptado: 24/04/2017

\begin{abstract}
Resumen
Presentamos una propuesta de ficha metodológica para el análisis de la evidencialidad - entendida como la expresión de la fuente de información- en el discurso científico. La ficha ha sido aplicada a un estudio exploratorio de un corpus de artículos científicos (1799-1920). Combina variables formales, contextuales y semántico-pragmáticas. De esta manera, la ficha permite realizar un análisis exhaustivo de la evidencialidad, puesto que aporta información sobre qué clases de recursos se encuentran, cómo se insertan en el discurso y qué funciones realizan.
\end{abstract}

PALABRAS CLAVE: evidencialidad, metodología, ficha de análisis, discurso científico.

\begin{abstract}
This paper is a proposal of a methodology for analysing the category of evidentiality (understood as the expression of the source of information) in scientific discourse. The analysis sheet has been applied to an exploratory study of a corpus of scientific articles (1799-1920). It combines three types of variables: formal, contextual and semantic-pragmatic. It allows a comprehensive analysis of evidentiality, since it provides information on what kinds of expressions are found, how they are inserted in the discourse and what functions they perform.

KEY WORDS: evidentiality, methodology, analysis sheet, scientific discourse.
\end{abstract}

1 Este trabajo ha contado con el apoyo del proyecto Proyecto Es.Vag.Atenuación "La atenuación pragmática en su variación genérica: géneros discursivos escritos y orales en el español de España y América" (http://esvaratenuacion.es), Proyecto MINECO FFI2016-75249-P.

Para citar este artículo / To cite this article: Kotwica, Dorota (2018): Propuesta metodológica para el análisis de la evidencialidad en el discurso científico. García Ramón, Amparo y Soler Bonafont, María Amparo (Eds.): ELUA: Estudios de atenuación en el discurso, Anexo IV, págs. 197-212.

Enlace / Link: http://dx.doi.org/10.14198/ELUA2018.Anexo4.11 


\section{INTRODUCCIÓN}

En los últimos años ha ido aumentando el interés de la lingüística sobre la pertinencia de la categoría de la evidencialidad en lenguas europeas no evidenciales, entre ellas, el español. Gracias a estos esfuerzos, los límites de esta categoría quedan cada vez mejor definidos, así como sus características y las funciones que tiene el uso de evidenciales en distintos géneros discursivos.

No obstante, en la actualidad, no existe un consenso entre los estudiosos sobre cómo se reconoce la evidencialidad en el discurso y qué parámetros deben aplicarse a la hora de analizar su modo de expresión en distintos géneros. ¿Qué debe entenderse exactamente como expresión de la evidencialidad en términos de elementos lingüísticos concretos? ¿Qué variables deberían incluirse en el análisis de las funciones de esta categoría en el discurso? ¿Qué factores específicos de análisis hay que tener en cuenta a la hora de estudiar evidencialidad en géneros científicos? Estas son las preguntas que nos planteamos cuando emprendíamos el estudio sobre la evidencialidad en artículos científicos del siglo XIX y de los inicios del siglo XX. Vimos necesario establecer criterios objetivos de estudio y confeccionar una ficha de variables de análisis aplicable al corpus de textos históricos.

El hecho de realizar un intenso análisis de corpus supuso la oportunidad de ensayar la ficha que habíamos diseñado previamente, tras la revisión del aparato teórico. La actualizamos y la perfilamos de tal manera que pudiera ser útil también para otros analistas y otros corpus discursivos, con pequeños ajustes.

En este artículo exponemos una nómina razonada y organizada de variables para el análisis de la expresión de la evidencialidad en el discurso científico. Justificamos la selección de las variables y aportamos ejemplos de algunos de los valores propuestos en la ficha. Los ejemplos proceden del corpus mencionado anteriormente, en el que se han mantenido las grafías del texto original.

\section{CONSIDERACIONES PREVIAS}

La ficha de análisis que se expone en este artículo se diseñó para una investigación centrada en examinar la evolución del género del artículo científico entre 1799 y 1920 a través de la expresión de la evidencialidad. Por lo tanto, se trata de una ficha que pretende ser útil tanto para un análisis de la expresión de la evidencialidad en el español en general como, más específicamente, la expresión de esta categoría en el discurso científico. La estructura general de la ficha se ha inspirado en la ficha para el análisis de la atenuación del grupo investigador Es.Var.Atenuación y Val.Es.Co. (Albelda, Briz, Cestero, Kotwica y Villalba 2014).

En el diseño de las variables del análisis, hemos tenido presentes los avances en el estudio de la evidencialidad y de su relación con el discurso científico. Si bien en muchas ocasiones remitiremos al marco teórico para justificar nuestras decisiones metodológicas, prescindiremos en esta ocasión de ahondar en la discusión teórica sobre la evidencialidad. Nuestro objetivo en este trabajo es distinto y mucho más aplicado, esto es, pretendemos proponer soluciones prácticas para estudiar la expresión de la evidencialidad en el discurso y en una lengua tipológicamente no evidencial, en la que, por tanto, la evidencialidad no está gramaticalizada. En este sentido, el principal factor condicionante del análisis fue la definición de la evidencialidad que se había adoptado. No se trata de una definición novedosa que rompa con lo establecido hasta ahora, sino, más bien, de una definición con la que 
hacemos patente nuestra posición respecto a las principales controversias que existe en la bibliografía acerca de la evidencialidad (por ejemplo, qué tipo de categoría es y qué clase de elementos abarca). Asimismo, es una definición que ofrece pautas prácticas sobre qué clase de elementos lingüísticos consideramos como recursos evidenciales. Pone, por tanto, las bases para el estudio del corpus. Reproducimos esta definición a continuación:

La EVIDENCIALIDAD es una categoría semántico-funcional responsable de aportar fuentes de información y modo de conocimiento (definición categorial amplia) con los que cuenta el hablante. Su relación con la modalidad epistémica no es estable, por lo que la evidencialidad puede estudiarse de forma independiente a la modalidad (definición conceptual estrecha). En español, la evidencialidad se expresa por medio de diferentes categorías formales de la lengua que conforman un conjunto abierto de recursos evidenciales.

RECURSOS EVIDENCIALES son elementos lingüísticos explícitos que señalan el modo de obtener la información y/o su fuente. Son a menudo elementos polisémicos que pueden funcionar como evidenciales en contextos factuales y, normalmente, cuando su ámbito se puede definir como ámbito proposicional. (Kotwica 2016: 189)

Este planteamiento implica que el punto de partida para el análisis del corpus sea el recurso evidencial, definido como elemento o construcción de la lengua que cumple la función evidencial en su contexto. Asimismo, para el análisis es esencial tomar en consideración el "contexto interaccional concreto" (Albelda et alii 2014) dentro del que se evalúan los valores evidenciales y pragmáticos de una construcción.

Es necesario tener en cuenta que, en español, un elemento de la lengua a veces es considerado un recurso evidencial y otras, no. Además, dependiendo del contexto, puede transmitir significados evidenciales distintos y cumplir funciones diferentes. Solo si existiera una clara asociación entre determinados elementos lingüísticos y el tipo de significado evidencial, el análisis del corpus podría realizarse de una manera más automatizada. Puesto que en español esto no es posible, resulta necesario analizar manualmente cada ocurrencia y evitando el prejuicio de que un elemento lingüístico siempre exprese los mismos valores evidenciales o que siempre realice la misma función pragmática.

Un ejemplo muy claro de lo complejo que resulta el análisis de los recursos evidenciales en español son los diferentes usos del verbo ver. Siguiendo nuestra definición, trataríamos como evidencial su uso restringido a la percepción del hablante y en un contexto factual, como por ejemplo en (1).

(1) He visto que los estudiantes abandonaron el edificio de la facultad después de terminar las clases.

Por el contrario, no lo incluiríamos en la nómina de los evidenciales si introduce visión de otras personas (2), si opera sobre un simple ámbito nominal (3) o si se encuentra dentro de una construcción no factual (4).

(2) El profesor vio que los estudiantes abandonaron el edificio de la facultad después de terminar las clases.

(3) He visto a los estudiantes. 
(4) Si hubiera estado allí, hubiera visto que los estudiantes abandonaron el edificio de la facultad después de terminar las clases.

Además, dependiendo del contexto, un mismo recurso puede transmitir significados evidenciales diferentes. El verbo ver también se presta como un caso ejemplar para esta realidad, puesto que, por su carácter polisémico, puede introducir el significado de la visión física o de la visión cognitiva (Whitt 2010a, Sweetser 1990). El primer significado se relaciona con la evidencialidad directa visual, mientras que el segundo, con la evidencialidad inferencial.

Teniendo en cuenta el doble interés del estudio, esto es, por un lado, la evidencialidad $\mathrm{y}$, por el otro, su relación con la evolución del género del artículo científico, incluimos en la ficha tres tipos de variables que en conjunto ofrecen una panorámica bastante completa de la interacción de los elementos concretos de la lengua, el contexto en el que se integran y su funcionamiento pragmático y discursivo.

- Variables que analizan el comportamiento semántico-pragmático y el funcionamiento discursivo de recursos evidenciales.

- Variables relativas al contexto en el que se insertan los recursos evidenciales.

- Variables relativas a la forma de recursos evidenciales.

En nuestro análisis, hemos realizado pruebas estadísticas utilizando las variables que se expondrán a continuación, todas de ellas, nominales. Cada variable cuenta con una serie de valores que permiten clasificar los elementos analizados. Dichos valores fueron establecidos teniendo en cuenta el marco teórico, así como los resultados del estudio piloto del corpus. Por tanto, dependiendo de los intereses del investigador y del tipo de corpus analizado, algunos valores podrían resultar irrelevantes o bien podría ser necesario introducir valores adicionales.

Finalmente, cabe advertir que no todas las variables tienen el mismo alcance, es decir, no todas sirven para analizar todos los casos posibles del corpus. Por ejemplo, sugerimos algunas variables adicionales para una mejor descripción de recursos de evidencialidad reportativa. Clasifican los recursos reportativos desde la perspectiva del tipo de la fuente indirecta de información que estos introducen. Por lo tanto, no sirven para describir recursos de otros tipos de evidencialidad, puesto que no existe en ellos una dimensión de fuente indirecta de información. Por su alcance, limitado a tan solo un grupo de casos en el corpus, se trata de variables que no son relevantes para un análisis general del corpus, pero sí aportan datos interesantes sobre un tipo de evidencialidad en el discurso científico.

\section{VARIABLES SEMÁNTICO-PRAGMÁTICAS}

En primer lugar, presentamos las variables que analizan el significado y las funciones de un recurso evidencial.

VARIABLE 1. TIPO DE SIGNIFICADO EVIDENCIAL

Valores: evidencialidad visual, inferencia ${ }_{1}$ inferencia ${ }_{2}$, evidencialidad reportativa

En esta variable se analiza el significado evidencial que un recurso expresa en su contexto. La clasificación de los valores se basa en la bibliografía e incluye los tipos de 
evidencialidad tradicionalmente considerados en los trabajos sobre este tema (Willet 1988, Aikhenvald 2004, Bermúdez 2005, Squartini 2008, Plungian 2010, entre otros), ligeramente adaptados a la realidad de los artículos científicos.

La evidencialidad visual se refiere a los datos obtenidos a través de la observación directa y personal del autor (5):

(5) Durante los meses de Agosto y Setiembre, en que suele abundar en El Escorial la Phylloxera coccinea, la he visto cubrir el envés de las hojas del Quercus Toza y lusitanica con preferencia á las demás (...). (12-1887-MemoRAC)

La inferencia ${ }_{1}(6)$ es aquella que el hablante formula a partir de los estímulos percibidos por los sentidos (normalmente, la visión) o a partir de otros datos concretos y explícitos en el contexto. Es, por tanto, fruto del razonamiento que el hablante desarrolla a partir de estímulos físicos y concretos.

(6) De mis comprobaciones resulta, hasta ahora, que se obtiene riqueza de neurofibrillas con el nitrato de plata, á la dosis indicada, en cuarenta y ocho horas con tubo de radio de 3.000 actividades, y en veinticuatro horas con tubo de 10.000, y en menos tiempo con el de 100.000. (14-1905-RevRAC)

Por su parte, la inferencia 2 (7) se aplica al razonamiento lógico o basado en los conocimientos previos del hablante, pero sin una base perceptiva apreciable en el contexto. La diferencia entre los dos tipos de inferencias reside en que la inferencia ${ }_{2}$ se formula con una mayor implicación de componentes de razonamiento más generales o abstractos (conocimiento general del mundo, experiencias previas, etc.).

(7) Hermafroditismo en el que ambos conductos se abren ó terminan en una cloaca. Existen muchas variedades. ¿Cómo se hace la fecundacion? Es evidente que el animal puede fecundarse solo, pero sin embargo copula. ¿Para qué? Es probable que haya fecundacion recíproca en consecuencia de esta union. (6-1856-MemoRAC)

Finalmente, el valor evidencialidad reportativa es aquel procedente de fuentes externas, concretamente, del discurso de otras personas, bien oral (conversación, entrevista) o escrito (carta, artículo, libro). Como señalan numerosos estudios, las evidencias reportativas constituyen uno de los pilares del discurso científico contemporáneo (Chafe 1986, Hyland 2009, Dehkordi y Allami 2012, entre otros). Por ello, incluimos en el análisis tres variables adicionales específicamente diseñadas para clasificar estas evidencias con mayor precisión.

Variable adicional 1.1 Especificación de la fuente reportada (solo para recursos reportativos) Valores: conocimientos compartidos (no susceptibles a la especificación), no específica, semi-especifica, especifica

Teniendo en cuenta las características del discurso científico, una de las dimensiones más relevantes de los recursos evidenciales que se insertan en él es la precisión con la que 
se especifican las fuentes de información. De ahí surgen los sistemas complejos de citación en los que no se puede prescindir de ningún dato relativo a las obras citadas con tal de que sean fácilmente reconocibles y puedan ser consultadas por los lectores de un artículo.

En el campo de los estudios sobre la evidencialidad, esta variable se relaciona con la distinción entre reportativo/citativo (quotative, Aikhenvald 2004, Lampert y Lampert 2010) y con la relevancia de evidencias que hacen la fuente explícita en el contexto (Chafe 1986; Aikhenvald 2004, 2007; Dehkordi y Allami 2012).

Los valores que se incluyen son conocimientos compartidos (no susceptibles a la especificación), es decir, aquellos recursos reportativos que hacen referencia al conocimiento general o saberes compartidos, donde no se puede asignar una fuente concreta y donde, además, esta fuente resulta irrelevante. Aparte de este valor, proponemos tres valores más que conforman una simple escala de especificación de la fuente. El valor no específica se aplica a los recursos que señalan que existe una fuente concreta, pero no proporcionan datos para su identificación (8).

(8) El nervio óptico recibe también fibras centrífugas, como han indicado varios autores; en el ratón estas fibras parecen tener su arranque en células residentes en las regiones inferiores del tálamo, no lejos de la substancia nigra (13-1894-ASEHN)

El valor semi-especifica describe las fuentes identificadas de manera muy escueta y con poca precisión, por ejemplo, por medio de aportar solamente el apellido del científico responsable de una información (sin mencionar el trabajo concreto) (9).

(9) Las cenizas del Vesubio, como muy bien dice Longet, ¿no han sido transportadas únicamente por el intermedio del aire, no solo por toda la Italia, sino á parajes aún mas lejanos? (6-1856-MemoRAC)

Finalmente, el valor especifica designa los recursos reportativos en los que aparecen al menos dos piezas que describen la fuente (por ejemplo, apellidos y fecha de la publicación de la obra aludida; título del libro y número de página, etc.).

Esta variable resulta especialmente interesante para un análisis histórico como el nuestro, puesto que permite observar cómo se han ido consolidando las prácticas de citación de las fuentes externas durante la época. Asimismo, no solo aporta datos sobre la evidencialidad, sino también sobre la evolución de las características del género analizado.

Variable adicional 1.2 Tipo de fuente aludida (solo para los recursos reportativos) Valores: cientifica, no cientifica

Esta variable analiza qué tipos de autoridades se citan en el corpus, es decir, si se trata siempre de referencias a palabras o trabajos de otros científicos, o si aparecen también menciones de fuentes sin ninguna relación con la ciencia como testigos, ciudadanos de a pie, etc. Incluimos un ejemplo (10) de este segundo caso en el que clasificaríamos la fuente aludida como no científica, puesto que la voz reportada pertenece a los mineros de Chile que no pueden calificarse como pertenecientes a la comunidad científica. 
(10) Es muy freqüente ver en sus casas á las personas de ambos sexos con la xícara en la izquierda, y con la derecha el tubo que llevan á la boca, repitiendo esto veinte y mas veces cada dia. Lo mismo practican los mineros de Chile, y creen ser muy conducente á la salud el uso de este vegetal, llamado por excelencia yerba. (5-1803-AHN)

Variable adicional 1.3 Procedencia de la evidencia reportada (solo para los recursos reportativos)

Valores: fuente ajena, autocitación

Esta variable está relacionada con el interés que supone la autocitación para el análisis del discurso científico (Salager-Meyer 1999; Swales 2007 [1990]; Hyland 2001). Desde el punto de vista de la evidencialidad, la autocitación constituye un caso muy especial de evidencialidad reportativa, ya que, tal como indica el propio nombre y la definición clásica de esta última, se trata de aportar información de fuentes externas a uno mismo. Sin embargo, Estellés (2015) demuestra que, en algunos contextos, la autocitación se convierte en evidencia reportada, lo que se puede explicar por medio de la asignación de roles discursivos diferentes (a partir de la teoría de Ducrot 1986). Estellés (2015) analiza cómo los hablantes evocan en la conversación sus propias palabras enunciadas en otro momento. La mencionada autora argumenta que la identidad de la fuente (uno mismo) no invalida el carácter indirecto de este tipo de evidencias. Lo que ocurre en estos casos es que aparecen en el discurso dos roles discursivos asignados a la misma persona (el de locuteur y el de sujet parlant). Consideramos que algo muy similar ocurre con la autocitación en los trabajos científicos; son ejemplos de evidencialidad reportativa en los que la fuente que se aporta coincide con quien la cita. En el discurso científico, la autocitación implica remitir a textos ya existentes, con lo cual el propio autor puede incluso expresar su distanciamiento y crítica hacia ellos.

El ejemplo (11) contiene dos referencias. La primera remite a un trabajo de Van Gehuchten (fuente ajena) mientras que la segunda, al trabajo del autor del artículo citado (autocitación).

(11) Que del ganglio basal de los peces proceden fibras nerviosas que se incorporan al fascículo basal del cerebro anterior, es un hecho demostrado también recientemente por Van Gehuchten (4) y por nosotros (5). (13-1894-ASEHN).

Variable adicional 1.4 Tipo de observación (solo para los recursos de evidencialidad visual) Valores: ocular directo, a través de medios técnicos

El primer valor de esta variable es una observación ocular directa donde se ha obtenido la información a partir de una simple visión u observación más prolongada al ojo desnudo. El segundo, observación a través de medios técnicos, se refiere a la observación ayudada por herramientas científicas (microscopio, lupas, etc.) (12).

Se trata de una variable que puede ser relevante o no serlo, dependiendo de la disciplina de la que se trata. Si su interés para los textos de biología es indiscutible, podría ser cuestionado si el corpus analizado estuviera compuesto por textos de humanidades. Esta variable tiene también una relevancia especial dentro del contexto del estudio histórico del discurso científico, puesto que permite observar la expansión de nuevos métodos de la ciencia experimental en detrimento de la ciencia observacional. 
(12) Examinado por medio del microscopio se notan cuatro cosas: $1 .^{\circ}$ una parte fluida; $2 .^{\circ}$ glóbulos análogos á los glóbulos mucosos; $3 .^{\circ}$ gránulos elementales; y $4 .^{\circ}$ por encima innumerable cantidad de corpúsculos móviles filiformes, que son los que se denominan espermatozoidos, y á los cuales se les ha hecho desempeñar papeles muy diferentes en el mecanismo de la generacion. (6-1856-MemoRAC)

\section{VARIABLE 2. ACCESIBILIDAD DE LA EVIDENCIA}

Valores: compartida, no compartida

Esta variable se relaciona con la noción de accesibilidad, intersubjetividad y carácter compartido o no compartido de las evidencias (Nuyts 2001a, 2001b, 2012; Carretero 2002; Bermúdez 2005; Cornillie 2007, 2009; Whitt 2011; Marín-Arrese 2013; Alonso-Almeida 2015; Marcos Sánchez 2016).

La variable analiza si, en un contexto dado, un recurso evidencial se utiliza para indicar una evidencia compartida (es decir, la evidencia a la que pueden acceder más personas además del hablante) o no compartida (esto es, la evidencia a la que accede exclusivamente el hablante y nadie más). Puesto que tanto el carácter de evidencias en las que se basan los diferentes tipos de evidencialidad como el modo de acceso que se realiza a ellas son muy heterogéneos, consideramos que la accesibilidad debe medirse de manera diferente para cada subtipo evidencial.

Para determinar si una evidencia presenta o no carácter accesible hemos tenido en cuenta el contexto en el que aparece, la forma del recurso evidencial y el contexto general de las prácticas discursivas en el género analizado. En la Tabla 1 se resumen los criterios concretos para evaluar la accesibilidad de un recurso concreto, siempre partiendo, en primer lugar, del tipo de la evidencialidad que expresa.

\begin{tabular}{|c|c|c|}
\hline $\begin{array}{c}\text { TIPO DE LA } \\
\text { EVIDENCIALIDAD }\end{array}$ & $\begin{array}{l}\text { EVIDENCIA NO } \\
\text { COMPARTIDA }\end{array}$ & EVIDENCIA COMPARTIDA \\
\hline Visual & $\begin{array}{l}\text { Formas verbales que } \\
\text { indican la primera persona, } \\
\text { pronombres }\end{array}$ & $\begin{array}{l}\text { Formas verbales que indican visión u } \\
\text { observación compartida (impersonalizaciones, } \\
\text { generalizaciones) } \\
\text { Presencia de elementos visuales } \\
\begin{array}{l}\text { Descripción detallada de métodos de } \\
\text { observación } \\
\text { Observación de larga duración o general }\end{array}\end{array}$ \\
\hline Inferencia (1 y 2) & $\begin{array}{l}\text { Pautas sintácticas que } \\
\text { limitan el proceso de } \\
\text { razonamiento al escritor } \\
\text { La evidencia en la que se } \\
\text { basa el razonamiento no } \\
\text { está presente en el contexto }\end{array}$ & $\begin{array}{l}\text { Pautas sintácticas que no limitan el proceso de } \\
\text { razonamiento al escritor } \\
\text { La evidencia en la que se basa el razonamiento } \\
\text { está presente en el contexto }\end{array}$ \\
\hline Inferencia1 & & Elementos visuales \\
\hline Reportativa & $\begin{array}{l}\text { La fuente de la información } \\
\text { no se especifica }\end{array}$ & $\begin{array}{l}\text { La fuente de la evidencia se especifica } \\
\text { (referencias bibliográficas) } \\
\text { Marcadores de conocimientos comunes o saber } \\
\text { compartido }\end{array}$ \\
\hline
\end{tabular}

Tabla 1. Criterios para el análisis de la variable "accesibilidad"

(Kotwica en prensa, en prensa 1). 
Aportamos un ejemplo de evidencialidad visual compartida, en (13), donde el autor del texto remite a una observación impersonal (se observa que). En cambio, en el citado anteriormente ejemplo (5), encontramos un caso de observación personal y no compartida (he visto que)

(13) En las lombrices de tierra y en las de agua dulce se observa que, divididas en dos porciones, la anterior regenera el estremo caudal y la posterior el cefálico, aunque este es mas dificil y requiere como condicion esencial no quitar muchos anillos, en cuyo caso se reproduce en el espacio de diez á treinta dias la cabeza completa, á pesar de haber sustraido el ganglio cefálico. (6-1856-MemoRAC)

En cuanto a las inferencias, en el ejemplo (14) se observa un caso de inferencia no compartida, puesto que no se ofrecen explícitamente los argumentos que han llevado al científico a formular esta idea. Un ejemplo de inferencia ${ }_{2}$ compartida se aprecia en (7), donde por medio de aludir a algo evidente (es evidente que), el autor del texto sugiere que la información no le pertenece solo a él, sino que es disponible a más personas, incluidos los lectores del artículo.

(14) Este árbol se parece al antecedente en su altura y fructificación, y tal vez solo será su variedad, aunque en las hojas se nota bastante diferencia, que consiste en ser de color de ocre amarillo por el envés, ser angostas en la parte inmediata al peciolo, y anchas en la superior. (4-1801-AHN)

\section{VARIABLE 3. EVALUACIÓN}

Valores: no marcada, marca positiva, marca negativa

Esta variable pretende dar cuenta de la actitud de los autores de los artículos científicos hacia la fuente de información y/o la información introducida por esta fuente, si es que esta actitud (positiva o negativa) se ve claramente marcada en el texto.

El valor no marcada se aplica cuando no se percibe ninguna actitud concreta del autor. Este es el valor por defecto a no ser que en el contexto aparezcan explícitamente elementos de evaluación. La marca positiva se asigna cuando en el contexto aparecen expresiones de tono positivo y halagador, mientras que la marca negativa se asigna cuando en el contexto se expresa la disconformidad del autor del artículo con lo dicho (expresiones de crítica o de desacuerdo). En el ejemplo (9), citado anteriormente, se observan marcas de evaluación positiva (como muy bien dice Longet), mientras que en el siguiente ejemplo (15), se aprecian marcas de evaluación negativa hacia las fuentes indirecta citadas (subrayadas en el ejemplo).

(15) Mayor exageracion, no obstante, revelan los escritos de otros botánicos, tales como De Candolle, Adr. de Jussieu y Endlicher, que no han vacilado en tener por señales de tiempo sereno ó lluvioso la apertura ú oclusion de las hojas de la Porlieria, sin explicarse acerca de los diarios movimientos de las hojas, llegando el último á decir de ellas lo que de ninguna manera dijeron los autores españoles: coelo sereno expansis, instante pluvia contractis, es la terminante frase que Endlicher emplea con relación á esto, tanto en su Genera, como en su Enchiridion. (7-1861-MemoRAC) 


\section{VARIABLE 4. (PROVISIONAL) FUNCIÓN DEL RECURSO EVIDENCIAL} Valores: neutra, refuerzo (de la autoridad del cientifico), refuerzo ${ }_{1}$ (de una información), atenuación ${ }_{1}$ (autoprotectora), atenuación ${ }_{2}$ (protección del yo y del tú)

El empleo de los recursos evidenciales puede servir simplemente para objetivar la información e introducir fuentes de información, sin ninguna estrategia visible por parte del hablante (hablaríamos entonces del valor función neutra). No obstante, su uso en determinados contextos puede responder a fines pragmáticos o comunicativos (Albelda 2016).

Esta variable es la menos universal de todas las que se incluyen en esta ficha metodológica, puesto que el tipo de funciones pragmáticas desarrolladas por los recursos evidenciales depende directamente del tipo de discurso analizado (oral, escrito) e incluso puede variar entre géneros. Así, habría que adaptarla al tipo de género discursivo que en cada caso se analice.

En los artículos científicos, el uso estratégico de la evidencialidad a menudo está ligado al modo de presentar la evidencia como personal o impersonal (lo cual en muchos casos se relaciona con la VARIABLE 2. ACCESIBILIDAD). Por un lado, la impersonalización del discurso puede incidir en la presencia de valores atenuantes (Luukka y Marrkkanen 1997; Morales y Cassany 2008; Albelda y Cestero 2011). Por otro lado, de acuerdo con Hyland (2002), una presencia marcada del autor en el texto ayuda a hacer hincapié en la contribución del autor al campo de estudio, reforzar su autoridad y/o aumentar la aceptabilidad de las ideas expresadas. De todas formas, aunque las marcas de (im)personalización pueden ser de gran ayuda para reconocer una función, es imprescindible analizar cada elemento en su contexto interaccional concreto.

En aquellos recursos evidenciales que se han juzgado como atenuantes en su contexto, se pueden, asimismo, asignar unos valores de atenuación más concretos, de acuerdo con las funciones de atenuación (Albelda et alii 2014: 18). La atenuación autoprotectora puede darse cuando la imagen del científico se vea amenazada, por ejemplo, cuando este emite una opinión o un juicio demasiado tajante sobre alguna realidad ("Función 1. Velar por sí mismo autoprotegiéndose por lo dicho o por lo hecho, con un interés de ganar o no perder imagen. Autoprotección con imagen. Salvaguarda del yo. Curarse en salud." (Albelda et alii 2014: 18)). Un ejemplo de esta función sería el uso de un recurso evidencial accesible de inferencia ${ }_{1}$ para introducir conclusiones del científico derivadas de su estudio (16). Al utilizar una forma menos subjetiva y más accesible (se ve con claridad), el autor del trabajo no se compromete personalmente con lo afirmado.

(16) En todo caso se ve con claridad no tener la influencia del eclipse nada de extraordinario que se diferencie de la ejercida sobre las hojas de esta planta por una sombra de equivalente intensidad. (7-1861-MemoRAC)

Por su parte, el valor de protección del yo y del tú suele darse en un contexto de potencial conflicto con los intereses o diferencias de ideas con otros miembros de la comunidad científica o invasión al territorio de otros ("Función 2. Prevenir una posible amenaza a la imagen del otro o un posible obstáculo en la consecución de una meta. Prevención. Salvaguarda del yo y del tú" (Albelda et alii 2014: 20)). Entendemos que esta podría ser la razón por la que el autor del fragmento citado en (17) utiliza un recurso de carácter compartido (vemos que) para indicar una conclusión del estudio (esto es, que los ejemplares que estudió son de la 
misma especie que otros estudiados por otros científicos). Según se indica en el contexto, esta conclusión podría ser puesta en entredicho por otros expertos porque existen "ligeras diferencias" entre los resultados de varios estudios. Esto indica que el autor se encuentra en riesgo de cuestionar el trabajo de otros científicos o que podría iniciar una disputa científica, por lo que el hecho de utilizar un recurso de carácter compartido y no eminentemente personal en cierta manera matiza su afirmación.

(17) Vemos que, tanto por la longitud cuanto por la armadura de las ventosas, tamaño de sus ganchos, así como los del rostelo, nuestros ejemplares coinciden con los de Sweet y Meggitt, siendo genuinas D. Proglottina tipo y su var. dublanensis; difieren, no obstante, en el número de ganchos que forman las coronas, mayor en los nuestros que en las especies de ambos helmintólogos; teniendo en cuenta lo caedizos que son, pudiera muy bien tratarse de ejemplares incompletos; en cuanto a la armadura rostelar; así Sweet halla 44-50; Meggitt. 50 60; nosotros, 70-92, y Blanchard, 80-95; pero estas ligeras diferencias no justifican la validez de especies nuevas, como tampoco el número de proglotis en el estróbilo, que en todo caso, como Kowaleusky razonablemente hizo, debe ser carácter propio de una variedad. (17-1919-RevRAC)

Por su parte, el ejemplo (18) ilustra como una observación personal realizada por el científico se expone para reforzar la contribución del mismo.

(18) Mis observaciones sobre el asunto, verificadas en los bosques de la cordillera de Guadarrama, me han demostrado no ser necesario para la reproducción de la Phylloxera coccinea, las emigraciones alternadas, tales cuales nos las ha descrito nuestro ilustrado colega afidiófilo; pues por punto general he visto que, cuando semejantes emigraciones tienen lugar, obedecen, no á la precisión de pasar de una especie de planta á otra, sino á la dispersión natural de los enjambres de individuos que buscan estancias cómodas y ventajosas para satisfacer sus necesidades. (12-1887-MemoRAC)

\section{VARIABLES CONTEXTUALES}

En esta parte de la ficha metodológica se incluyen variables relativas a la estructura del texto científico en el que se integran los recursos evidenciales.

\section{VARIABLE 5. PARTE DE LA ESTRUCTURA DEL ARTÍCULO \\ Valores: introducción, estudio, conclusiones, nota al pie}

Se refiere a la parte formal de la estructura del trabajo científico en la que se insertan los recursos evidenciales. Esta variable no es en sí un factor directamente ligado a la evidencialidad, pero sí fundamental para su análisis discursivo. Para especificar las partes concretas que se consideran, hay que tener en cuenta el género textual analizado, la disciplina científica a la que pertenecen los textos y también el periodo histórico. Dependiendo de estos tres factores, los valores dentro de esta variable podrían ser introducción, revisión teórica, metodología, estudio de campo, conclusiones, etc.

En nuestro estudio trabajamos con el género del artículo científico del campo de las ciencias biológicas en el periodo entre 1799 y 1920. Este último factor resultó decisivo a la hora de establecer los valores de esta variable. Y es que los artículos analizados no presentaban 
una estructura organizada según la disposición a la que estamos familiarizados hoy en día. Así, optamos por reflejar en el análisis cuatro partes estructurales amplias, pero adecuadas para la descripción de los textos científicos de la época de estudio. Puesto que, en los artículos analizados, por lo común, estas partes no se señalizaban por medio de una división en apartados o subapartados, nos guiamos por la lectura de los artículos y el reconocimiento de funciones que cumplían determinados párrafos dentro de los mismos.

\section{VARIABLE 6. MARCAS DE PERSONA}

Valores: yo, nosotros, impersonal

Se trata de analizar la presencia en el contexto de determinadas formas personalizadas $\mathrm{y}$ despersonalizadas de pronombres personales, pronombres posesivos y formas verbales (García Negroni, 2008) que a veces también se observan en el propio recurso evidencial (véase ejemplo (18) mis observaciones me han demostrado, he visto), aunque no siempre es así. La presencia de este tipo de marcas no se puede valorar en el caso de recursos como referencias bibliográficas, adverbios, preposiciones, etc. y en estos casos se juzgarían marcas visibles en el entorno del recurso evidencial.

Esta variable permite observar relaciones entre distintos tipos de recursos evidenciales y el grado de subjetividad del texto. Asimismo, podría ayudar a determinar cómo ciertos tipos de fuentes de información se asocian con personas concretas.

\section{VARIABLES FORMALES}

La última parte de la ficha metodológica contiene las variables para el análisis formal de los elementos lingüísticos utilizados como recursos evidenciales. Esta parte del análisis permite recabar datos sobre cómo se expresa la evidencialidad en el género analizado. Los valores que se incluyen dentro de esta variable dependen directamente de la definición de la evidencialidad adoptada. Asimismo, incluimos tanto las categorías de la lengua que transmiten el significado evidencial como su significado básico como las que son elementos polisémicos que en determinados contextos funcionan como recursos evidenciales.

\section{VARIABLE 7. CLASIFICACIÓN DEL RECURSO}

Valores: recurso verbal, preposición o locución prepositiva, locución adverbial, adverbio en -mente, sustantivo en oración atributiva, adjetivo en oración atributiva, uso dislocado de un tiempo verbal, que citativo, frase/construcción, referencia, cita

Recurso verbal. Los verbos (de percepción, apariencia, deducción, inducción, pensamiento y del decir) son una de las categorías más amplias por medio de la cual se introducen significados evidenciales en español. Se trata de verbos en formas personales, impersonales y pasivas, así como cuando forman parte de las estructuras con conjunciones (según dice, como afirma, etc.). El reconocimiento del significado evidencial de un recurso verbal depende, en buen grado, de la semántica del verbo.

Preposición o locución prepositiva. Algunas preposiciones, en estructuras concretas, tienen la capacidad de introducir significados evidenciales (según X, para X). Con el mismo valor se pueden usar locuciones prepositivas como en opinión de, a juzgar por, en expresión de. 
Locución adverbial. Este valor abarca tanto las locuciones menos fijadas (en mi entender, a nuestro juicio, etc.) como las que, en el español actual, pueden ser consideradas marcadores o partículas discursivas ( $\sin d u d a$, al parecer, por lo visto, etc.). La decisión de agrupar todos estos casos dentro de un único valor se justifica por el carácter histórico del corpus y por la dificultad de evaluar, en cada caso, el grado de la posible gramaticalización de todas las construcciones.

Adverbios en -mente. Hemos reservado el valor adverbio para los usos evidenciales de los adverbios en -mente. La decisión de separar estos de las locuciones adverbiales está relacionada con nuestro interés de observar la presencia en los textos de los adverbios en -mente, puesto que, desde el punto de vista de la historia de la lengua, el siglo XIX se considera el momento de la expansión de este tipo de adverbios con el significado modalevidencial (Espinoza Elorza 2012).

Sustantivos en oraciones atributivas, adjetivos en oraciones atributivas. En esta clase de recursos son los elementos nominales o adjetivales los que se encargan de transmitir el significado evidencial, por ejemplo es hecho de la experiencia, es una cosa bien conocida, es sabido, etc.

Usos dislocados de tiempos verbales. A diferencia del valor recursos verbales, aquí la transmisión del significado evidencial no se hace por medio del valor semántico del verbo, sino desde una perspectiva morfológica y gracias al uso dislocado de un determinado tiempo verbal.

Que citativo. Se trata de casos donde que aparece como el único introductor de una información procedente de fuentes indirectas.

Construcción/frase. Este valor está reservado para para aquellos casos, en los que toda una frase o construcción compleja constituyen el recurso evidencial.

Referencias bibliográficas; citas. La presencia en esta ficha de estos dos valores viene justificada por el carácter del corpus analizado. Referencias o citas se incluyen como recursos evidenciales cuando aparecen solas, sin otro recurso introductorio como podría ser un verbo o una construcción con preposición, pues en el caso contrario analizaríamos el otro recurso introductorio como recurso evidencial y la referencia o cita como un modo de especificar la fuente indirecta.

Variable adicional 7.1 Formato de la referencia (solo para referencias)

Valores: insertadas, no insertadas

Esta variable adicional describe el formato de las referencias y sus valores se basan, principalmente, en la clasificación de Swales (2007 [1990]) en la que se hace una distinción básica entre las referencias insertadas y las no insertadas. En las referencias insertadas se incluye el nombre del autor citado, integrado en la sintaxis (sea en función de sujeto de la oración o en otras funciones). En cambio, las referencias no insertadas aparecen como elementos externos a la predicación. Un ejemplo claro de estas últimas es la aparición del nombre del autor y el año de la publicación entre paréntesis.

Variable adicional 7.2 Forma y voz del verbo (solo para recursos verbales) Valores: personal/activa, impersonal/pasiva

Esta variable resulta relevante porque los usos de formas pasivas o impersonales de algunos verbos inciden en su capacidad de expresar determinados tipos evidencialidad. Además, 
los verbos en formas que no apuntan a ninguna persona en concreto como responsable del acto de percepción o del acto de decir a menudo crean la impresión de objetividad o de carácter compartido de la evidencia. Esto último está relacionado con la accesibilidad de las evidencias o el carácter compartido de las mismas (Variable 2).

\section{VARIABLE 8. MODO DE INTEGRACIÓN}

Valores: integrado, no integrado

Esta variable indica si un recurso está integrado en la oración o si es un elemento externo a la predicación. Da pistas sobre el ámbito de actuación de los recursos evidenciales y permite registrar usos evidenciales de elementos parentéticos y comentarios externos.

Variable adicional 8.1 Posición (solo para los recursos no integrados)

Valores: anterior, intermedia o posterior

La variable informa sobre la posición de un recurso evidencial no integrado respecto al contenido para el que se aporta una evidencia. En combinación con la variable "modo de integración", esta permite estudiar con más detenimiento el comportamiento de recursos evidenciales con respecto a distintos ámbitos de actuación. El diseño de esta variable se ha inspirado en las convenciones utilizadas en el Diccionario de Partículas Discursivas del español (Briz et alii (coords.)).

\section{CONSIDERACIONES FINALES}

Tal como se desprende de la ficha metodológica presentada en este artículo, para el estudio de la evidencialidad en el discurso resultan relevantes tres dimensiones de análisis: la semántico-pragmática, que permite dar cuenta del funcionamiento de los evidenciales en el discurso; la contextual, que ayuda a reconocer como interactúan los evidenciales con la estructura del texto y la formal, necesaria para la descripción de los elementos lingüísticos que cumplen la función evidencial en la lengua. Consideramos que la combinación de las variables comprendidas entre estas tres dimensiones permite realizar un análisis muy completo de la expresión de la evidencialidad en distintos géneros discursivos españoles.

En el diseño de las variables, tuvimos en cuenta las particularidades de la expresión de la evidencialidad en español, especialmente, su dependencia del contexto y el carácter heterogéneo de los elementos lingüísticos que conforman la nómina de recursos evidenciales en esta lengua. Asimismo, adaptamos los parámetros del análisis al tipo de discurso al que esta se aplicaría. Si bien muchas variables serían válidas también para estudios dedicados a otros géneros discursivos, en algunos casos sería necesario realizar pequeños ajustes en su descripción. Las variables y los valores (por ejemplo, la variable especificación de la fuente o el valor referencia de la variable tipo de recurso evidencial) que resultan relevantes para el género del artículo científico no lo serían para otros géneros. Por lo tanto, creemos que las particularidades del tipo de discurso analizado constituyen el criterio principal a la hora de ajustar la metodología del análisis de la expresión de la evidencialidad. 


\section{Referencias bibliográficas}

Aikhenvald, A. Y. (2004). Evidentiality. Oxford: Oxford University Press.

Aikhenvald, A. Y. (2007). "Information source and evidentiality: what can we conclude?", Rivista di Linguistica, 19 (1), pp. 209-227.

Albelda, M. (2016). "Estableciendo límites entre la evidencialidad y la atenuación en español”. En González Ruiz, R., Izquierdo Alegría, D. y Ó. Loureda Lamas (eds.). La evidencialidad en español: teoría y descripción. Madrid: Iberoamericana/Vervuert, pp. 21-36.

Albelda, M. y A. M. Cestero (2011). "De nuevo, sobre los procedimientos de atenuación”, Español Actual, 96, pp. 121-55.

Albelda, M., Briz, A., Cestero, A. M., Kotwica, D. y C. Villalba Ibáñez (2014). "Ficha metodológica para el análisis pragmático de la atenuación en corpus discursivos del español. (ES.POR.ATENUACIÓN)", Oralia 17, pp. 7-62.

Alonso-Almeida, F. (2015): "The functions of seem and parecer in early medical writing", Discourse Studies, 17 (2), pp. 121-140.

Bermúdez, F. (2005). Evidencialidad. La codificación lingüística del punto de vista. Tesis doctoral, Stockholms Universitet.

Briz, A., Pons Bordería, S. y J. Portolés (coords.) (en línea). Diccionario de partículas discursivas del español: www.dpde.es (13-2-2017).

Carretero, M. (2002). "The influence of genre and register on epistemic modality in spoken English: a preliminary study", Estudios Ingleses de la Universidad Complutense, 10, pp. 11-41.

Chafe, W. L. (1986). "Evidentiality in English Conversation and Academic Writing". En Chafe, W. y J. Nichols (eds.). Evidentiality: The Linguistic Coding of Epistemology. Norwood: Ablex Publishing Corporation, pp. 261-272.

Cornillie, B. (2007). Evidentiality and Epistemic Modality in Spanish (Semi-) Auxiliaries. A CognitiveFunctional Approach. Berlin/New York: Mouton de Gruyter.

Cornillie, B. (2009). "Evidentiality and epistemic modality. On the close relationship between two different categories", Functions of Language, 16 (1), pp. 44-62.

Dehkordi M. E. y H. Allami (2012). "Evidentiality in Academic Writing", Theory and Practice in Language Studies, 2 (9), pp. 1895-1904.

Diewald, G. y E. Smirnova (eds.) (2010). Linguistic Realization of Evidentiality in European Languages. Berlín/Nueva York: De Gruyter.

Ducrot, O. (1986). El decir y lo dicho. Polifonía de la enunciación. Barcelona/Buenos Aires: Paidós.

Estellés, M. (2015). "Expressing evidentiality through prosody? Prosodic voicing in reported speech in Spanish colloquial conversations", Journal of Pragmatics, 85, pp. 138-154.

García Negroni, M. M. (2008). "Subjetividad y discurso científico-académico. Acerca de algunas manifestaciones de la subjetividad en el artículo de investigación en español", Revista Signos 41 (66), pp. 5-31.

González Ruiz, R., Izquierdo Alegría, D. y O. Loureda Lamas (eds.) (2016). La evidencialidad en español: teoría y descripción. Madrid: Iberoamericana/Vervuert.

Hyland, K. (2001). ““"Humble servants of the discipline?" Self-mention in research articles”, English for Scientific Purposes, 20, pp. 207-226.

Hyland, K. (2002). "Authority and invisibility: authorial identity in academic writing", Journal of Pragmatics, 34, pp. 1091-112.

Hyland, K. (2009). Academic Discourse. London: Continuum International Publishing.

Kotwica, D. (2016). Evolución del género artículo científico en español (1799-1920) a la luz de la expresión de la evidencialidad. Tesis doctoral, Universidad de Valencia.

Kotwica, D. (en prensa). "Verbos de percepción evidenciales en artículos científicos del siglo XIX: Funciones pragmático-retóricas". 
Kotwica, D. (en prensa 1). "Exploring evidentiality in Spanish Biology articles (1850-1920). Intersubjectivity and accessibility of evidences".

Lampert G. y M. Lampert (2010). "Where does evidentiality reside? Notes on (alleged) limiting cases: seem and be like", STUF - Language Typology and Universals Sprachtypologie und Universalienforschung, 63 (4), pp. 308-321.

Luukka, M. R. y R. Marrkkanen (1997). "Impersonalization as a Form of Hedging”. En Markkanen, R. y H. Schröder (eds.). Research in Text Theory. Hedging and Discourse. Approaches to the Analysis of a Pragmatic Phenomenon in Academic Texts. Berlin/Boston: De Gruyter, pp. 168-187.

Marcos Sánchez, M. (2016). "Subjetividad e intersubjetividad en los marcadores evidenciales formados por el verbo de percepción visual ver en español”. En González Ruiz, R., Izquierdo Alegría, D. y Ó. Loureda Lamas (eds.). La evidencialidad en español: teoría y descripción. Madrid: Iberoamericana/Vervuert, pp. 153-177.

Marín-Arrese, J. I. (2013). "Stancetaking and inter/subjectivity in the Iraq Inquiry: Blair vs. Brown". En Marín-Arrese, J. I., Carretero, M. y J. Arús Hita (eds.). English Modality, Berlin/Boston: De Gruyter, pp. 411-445.

Morales, O. y D. Cassany (2008). "Interpretaciones de la atenuación en artículos de revisión de Odontología”, Signos, 41, pp. 299-328.

Nuyts, J. (2001a). "Subjectivity as an evidential dimension in epistemic modal expressions", Journal of Pragmatics, 33, pp. 383-400.

Nuyts, J. (2001b). Epistemic Modality, Language, and Conceptualization: A Cognitive-Pragmatic Perspective. Philadelphia: John Benjamins.

Nuyts, J. (2012). "Notions of (inter)subjectivity", English Text Construction, 5 (1), pp. 53-76.

Plungian, V. (2010). "Types of verbal evidentiality marking: an overview". En Diewald, G. y E. Smirnova (eds.). Linguistic Realization of Evidentiality in European Languaes, Berlín/Nueva York: De Gruyter, pp. 15-58.

Salager-Meyer, F. (1999). "Referential behaviour in scientific writing: a diachronic study (18101995)", English for Specific Purposes, 18(3), pp. 279-305.

Squartini, M. (2008). "Lexical vs. grammatical evidentiality in French and Italian”, Linguistics 46:5, pp. 917-947.

Swales, J. M. (ed.) (2007 [1990]). Genre Analysis. English in academic and research settings. Cambridge: Cambridge University Press.

Sweetser E. (1990). From etymology to pragmatics: Metaphorical and cultural aspects of semantic structure. Cambridge: Cambridge University Press.

Whitt, R. (2010a). "Evidentiality, polysemy, and the verbs of perception in English and German". In Diewald, Gabriele and Elena Smirnova (eds.) Linguistic realization of evidentiality in European Languages, pp. 255-284.

Whitt, R. (2011). "(Inter)Subjectivity and evidential perception verbs in English and German”, Journal of Pragmatics, 43, pp. 347-360.

Willet, T. (1988). "A cross-Linguistic Survey of the grammaticization of evidentiality, Studies in Language 12 (1), pp. 51-97. 